\title{
The renewed limit theorems for the discrete-time branching process and its conditioned limiting law interpretation
}

\author{
Azam Abdurakhimovich Imomov
}

State Testing Center, Under the Cabinet of Ministers of the Republic of Uzbekistan and Institute of Mathematics at the National University of Uzbekistan.

Received: 3 May 2016, Accepted: 10 August 2016

Published online: 12 November 2016.

\begin{abstract}
Our principal aim is to observe the Markov discrete-time process of population growth with long-living trajectory. First we study asymptotical decay of generating function of Galton-Watson process for all cases as the Basic Lemma. Afterwards we get a Differential analogue of the Basic Lemma. This Lemma plays main role in our discussions throughout the paper. Hereupon we improve and supplement classical results concerning Galton-Watson process. Further we investigate properties of the population process so called Q-process. In particular we obtain a joint limit law of Q-process and its total state. And also we prove the analogue of Law of large numbers and the Central limit theorem for total state of Q-process.
\end{abstract}

Keywords: Branching process, transition function, Q-process, invariant measures, ergodic chain, total states, joint distribution, limit theorem.

\section{Introduction}

The Galton-Watson branching process (GWP) is a famous classical model for population growth. Although this process is well-investigated but it seems to be wholesome to deeper discuss and improve some famed facts from classical theory of GWP. In first half part of the paper, Sections 2 and 3, we will develop discrete-time analogues of Theorems from the paper of the author [5]. These results we will exploit in subsequent sections to discuss properties of so-called Q-process as GWP with infinite-living trajectory.

Let a random function $Z_{n}$ denotes the successive population size in the GWP at the moment $n \in \mathbb{N}_{0}$, where; $\mathbb{N}_{0}=\{0\} \cup \mathbb{N}$ and $\mathbb{N}=\{1,2, \ldots\}$. The state sequence $\left\{Z_{n}, n \in \mathbb{N}_{0}\right\}$ can be expressed in the form of

$$
Z_{n+1}=\xi_{n 1}+\xi_{n 2}+\cdots+\xi_{n Z_{n}}
$$

where $\xi_{n k}, n, k \in \mathbb{N}_{0}$, are independent variables with general offspring law $p_{k}:=\mathbb{P}\left\{\xi_{11}=k\right\}$. They are interpreted as a number of descendants of $k$-th individual in $n$-th generation. Owing to our assumption $\left\{Z_{n}, n \in \mathbb{N}_{0}\right\}$ is a homogeneous Markov chain with state space $\mathscr{S} \subset \mathbb{N}_{0}$ and transition functions

$$
P_{i j}:=\mathbb{P}\left\{Z_{n+1}=j \mid Z_{n}=i\right\}=\sum_{k_{1}+\cdots+k_{i}=j} p_{k_{1}} \cdot p_{k_{2}} \cdots p_{k_{i}}
$$

for any $i, j \in \mathscr{S}$, where $p_{j}=P_{1 j}$ and $\sum_{j \in \mathscr{S}} p_{j}=1$. And on the contrary, any chain satisfying to property (1) represents GWP with the evolution law $\left\{p_{k}, k \in \mathscr{S}\right\}$. Thus, our GWP is completely defined by setting the distribution $\left\{p_{k}\right\}$; see [1, 
pp.1-2], [9, p.19]. From now on we will assume that $p_{k} \neq 1$ and $p_{0}>0, p_{0}+p_{1}<1$.

A probability generating function (GF) and its iterations is important analytical tool in researching of properties of GWP. Let

$$
F(s)=\sum_{k \in \mathscr{S}} p_{k} s^{k}, \quad \text { for } 0 \leq s<1
$$

Obviously that $A:=\mathbb{E} \xi_{11}=F^{\prime}(s \uparrow 1)$ denotes the mean per capita number of offspring provided the series $\sum_{k \in \mathscr{S}} k p_{k}$ is finite. Owing to homogeneous Markovian nature transition functions

$$
P_{i j}(n):=\mathbb{P}_{i}\left\{Z_{n}=j\right\}=\mathbb{P}\left\{Z_{n+r}=j \mid Z_{r}=i\right\}, \quad \text { for any } r \in \mathbb{N}_{0}
$$

satisfy to the Kolmogorov-Chapman equation

$$
P_{i j}(n+1)=\sum_{k \in \mathscr{S}} P_{i k}(n) P_{k j}, \quad \text { for } i, j \in \mathscr{S}
$$

Hence

$$
\mathbb{E}_{i} s^{Z_{n}}:=\sum_{j \in \mathscr{S}} P_{i j}(n) s^{j}=\left[F_{n}(s)\right]^{i}
$$

where GF $F_{n}(s)=\mathbb{E}_{1} s^{Z_{n}}$ is $n$-fold functional iteration of $F(s)$; see [3, pp.16-17].

Throughout this paper we write $\mathbb{E}$ and $\mathbb{P}$ instead of $\mathbb{E}_{1}$ and $\mathbb{P}_{1}$ respectively.

It follows from (2) that $\mathbb{E} Z_{n}=A^{n}$. The GWP is classified as sub-critical, critical and supercritical, if $A<1, A=1$ and $A>1$, accordingly.

The event $\left\{Z_{n}=0\right\}$ is a simple absorbing state for any GWP. The limit $q=\lim _{n \rightarrow \infty} P_{10}(n)$ denotes the process starting from one individual eventually will be lost and called the extinction probability of GWP. It is the least non-negative root of $F(q)=q \leq 1$ and that $q=1$ if the process is non-supercritical. Moreover the convergence $\lim _{n \rightarrow \infty} F_{n}(s)=q$ holds uniformly for $0 \leq s \leq r<1$. An assertion describing decrease speed of the function $R_{n}(s):=q-F_{n}(s)$, due to its importance, is called the Basic Lemma (in fact this name is usually used for the critical situation).

In Section 2 we follow on intentions of papers [7] and [5] and prove an assertion about asymptote of the function $R_{n}^{\prime}(s)$ as Differential Analogue of Basic Lemma. This simple assertion (and its corollaries, Theorem 1 and 2) will lays on the basis of our reasoning in Section 3.

We start the Section 3 with recalling the Lemma 3 proved in [1, p.15]. Until the Theorem 6 we study ergodic property of transition functions $\left\{P_{i j}(n)\right\}$, having carried out the comparative analysis of known results. We discuss a role of $\mu_{j}=\lim _{n \rightarrow \infty} P_{1 j}(n) / P_{11}(n)$ qua the invariant measures and seek an analytical form of GF $\mathscr{M}(s)=\sum_{j \in \mathscr{S}} \mu_{j} s^{j}$ and also we discuss $\mathscr{R}$-classification of GWP. Further consider the variable $\mathscr{H}$ denoting an extinction time of GWP, that is $\mathscr{H}=\min \left\{n: Z_{n}=0\right\}$. An asymptote of $\mathbb{P}\{\mathscr{H}=n\}$ has been studied in [12] and [20]. The event $\{n<\mathscr{H}<\infty\}$ represents a condition of $\left\{Z_{n} \neq 0\right\}$ at the moment $n$ and $\left\{Z_{n+k}=0\right\}$ for some $k \in \mathbb{N}$. By the extinction theorem $\mathbb{P}_{i}\{\mathscr{H}<\infty\}=q^{i}$. Therefore in non-supercritical case $\mathbb{P}_{i}\{n<\mathscr{H}<\infty\} \equiv \mathbb{P}_{i}\{\mathscr{H}>n\} \rightarrow 0$. Hence, $Z_{n} \rightarrow 0$ with probability one, so in these cases the process will eventually die out. We also consider a conditional distribution

$$
\mathbb{P}_{i}^{\mathscr{H}(n)}\{*\}:=\mathbb{P}_{i}\{* \mid n<\mathscr{H}<\infty\} .
$$


in the section. The classical limit theorems state that if $q>0$ then under certain moment assumptions the limit $\widetilde{P}_{i j}(n):=\mathbb{P}_{i}^{\mathscr{H}(n)}\left\{Z_{n}=j\right\}$ exists always; see [1, p.16]. In particular, Seneta [19] has proved that if $A \neq 1$ then the set $\left\{v_{j}:=\lim _{n \rightarrow \infty} \widetilde{P}_{1 j}(n)\right\}$ represents a probability distribution and, limiting GF $\mathscr{V}(s)=\sum_{j \in \mathscr{S}} v_{j} s^{j}$ satisfies to Schroeder equation

$$
1-\mathscr{V}\left(\frac{F(q s)}{q}\right)=\beta \cdot[1-\mathscr{V}(s)]
$$

where $\beta=F^{\prime}(q)$. The equation (3) determines an invariant property of numbers $\left\{v_{j}\right\}$ with respect to the transition functions $\left\{\widetilde{P}_{1 j}(n)\right\}$ and, the set $\left\{v_{j}\right\}$ is called $\mathscr{R}$-invariant measure with parameter $\mathscr{R}=\beta^{-1}$; see [17]. In the critical case we know the Yaglom theorem about a convergence of conditional distribution of $2 Z_{n} / F^{\prime \prime}(1) n$ given that $\{\mathscr{H}>n\}$ to the standard exponential law. In the end of the Section we investigate an ergodic property of probabilities $\widetilde{P}_{i j}(n)$ and we refine above mentioned result of Seneta, having explicit form of $\mathscr{V}(s)$.

More interesting phenomenon arises if we observe the limit of $\mathbb{P}_{i}^{\mathscr{H}(n+k)}\{*\}$ letting $k \rightarrow \infty$ and fixed $n \in \mathbb{N}$. In Section 4 we observe the conditioned limit $\lim _{k \rightarrow \infty} \mathbb{P}_{i}^{\mathscr{H}(n+k)}\left\{Z_{n}=j\right\}$ which represents an honest probability measures $\mathbf{Q}=\left\{\mathscr{Q}_{i j}(n)\right\}$ and defines homogeneous Markov chain called the Q-process. Let $W_{n}$ be the state at the moment $n \in \mathbb{N}$ in Q-Process. Then $W_{0} \stackrel{d}{=} Z_{0}$ and $\mathbb{P}_{i}\left\{W_{n}=j\right\}=\mathscr{Q}_{i j}(n)$. The Q-process was considered first by Lamperti and Ney [15]; see, also [1, pp.56-60]. Some properties of it were discussed by Pakes [17], [18], and in [6], [8]. The considerable part of the paper of Klebaner, Rösler and Sagitov [13] is devoted to discussion of this process from the viewpoint of branching transformation called the Lamperti-Ney transformation. Continuous-time analogue of Q-process was considered by the author [7].

Section 5 is devoted to classification properties of Markov chain $\left\{W_{n}, n \in \mathbb{N}\right\}$. Unlike of GWP the Q-process is classified on two types depending on value of positive parameter $\beta$. It is positive-recurrent if $\beta<1$ is transient if $\beta=1$. The set $\left\{v_{j}:=\lim _{n \rightarrow \infty} \mathscr{Q}_{i j}(n) / \mathscr{Q}_{i 1}(n)\right\}$ is an invariant measure for Q-process. The section studies properties of the invariant measure.

Sections 6 and 7 are devoted to examine of structure and long-time behaviors of the total state $S_{n}=\sum_{k=0}^{n-1} W_{k}$ in Q-process until time $n$. First we consider the joint distribution of the cumulative process $\left\{W_{n}, S_{n}\right\}$. As a result of calculation we will know that in case of $\beta<1$ the variables $W_{n}$ and $S_{n}$ appear asymptotically not dependent. But in the case $\beta=1$ we state that under certain conditions the normalized cumulative process $\left(W_{n} / \mathbb{E} W_{n} ; S_{n} / \mathbb{E} S_{n}\right)$ weakly converges to the two-dimensional random vector having a finite distribution. Comparing results of old researches we note that in case of $\beta=1$ the properties of $S_{n}$ essentially differ from properties of the total progeny of simple GWP. In this connection we refer the reader to [2], [10] and [11] in which an interpretation and properties of total progeny of GWP in various contexts was investigated. In case of $\beta<1$, in accordance with the asymptotic independence property of $W_{n}$ and $S_{n}$ we seek a limiting law of $S_{n}$ separately. So in Section 7 we state and prove an analogue of Law of Large Numbers and the Central Limit Theorem for $S_{n}$.

\section{Basic lemma and its differential analogue}

In this section we observe an asymptotic property of the function $R_{n}(s):=q-F_{n}(s)$ and its derivative. In the critical situation an asymptotic explicit expansion of this function is known from the classical literature which is given in the formula (4) below.

Let $A \neq 1$. First we consider $s \in[0 ; q)$. The mean value theorem gives

$$
R_{n+1}(s)=F^{\prime}\left(\xi_{n}(s)\right) R_{n}(s)
$$


where $\xi_{n}(s)=q-\theta R_{n}(s), 0<\theta<1$. We see that $\xi_{n}(s)<q$. Since the GF and its derivatives are monotonically nondecreasing then consecutive application of (4) leads $R_{n}(s)<q \beta^{n}$. Collecting last finding and seeing that $\beta<1$ we write following inequalities:

$$
F^{(k)}\left(q\left(1-\beta^{n}\right)\right)<F^{(k)}\left(\xi_{n}(s)\right)<F^{(k)}(q), \text { for } k=1,2 .
$$

In (5) the top index means derivative of a corresponding order. Considering together representation (4) and inequalities (5) we take relations

$$
\frac{R_{n+1}(s)}{\beta}<R_{n}(s)<\frac{R_{n+1}(s)}{F^{\prime}\left(q\left(1-\beta^{n}\right)\right)} \text {. }
$$

In turn, by Taylor formula and the iteration for $F(s)$ we have expansion

$$
R_{n+1}(s)=\beta R_{n}(s)-\frac{F^{\prime \prime}\left(\xi_{n}(s)\right)}{2} R_{n}^{2}(s), \quad \text { as } n \rightarrow \infty
$$

where and throughout this section $\xi_{n}(s)$ is such for which are satisfied relations (5). Assertions (5)-(7) yield:

$$
\frac{F^{\prime \prime}\left(q\left(1-\beta^{n}\right)\right)}{2 \beta}<\frac{\beta}{R_{n+1}(s)}-\frac{1}{R_{n}(s)}<\frac{F^{\prime \prime}(q)}{2 F^{\prime}\left(q\left(1-\beta^{n}\right)\right)} .
$$

Repeated application of (8) leads us to the following:

$$
\frac{1}{2 \beta} \sum_{k=0}^{n-1} F^{\prime \prime}\left(q\left(1-\beta^{k}\right)\right) \beta^{k}<\frac{\beta^{n}}{R_{n}(s)}-\frac{1}{q-s}<\frac{F^{\prime \prime}(q)}{2} \sum_{k=0}^{n-1} \frac{\beta^{k}}{F^{\prime}\left(q\left(1-\beta^{k}\right)\right)} .
$$

Taking limit as $n \rightarrow \infty$ from here we have estimation

$$
\frac{\Delta_{1}}{2} \leq \lim _{n \rightarrow \infty}\left[\frac{\beta^{n}}{R_{n}(s)}-\frac{1}{q-s}\right] \leq \frac{\Delta_{2}}{2}
$$

where

$$
\Delta_{1}:=\sum_{k \in \mathbb{N}_{0}} \frac{F^{\prime \prime}\left(q\left(1-\beta^{k}\right)\right)}{\beta} \beta^{k} \quad \text { and } \quad \Delta_{2}:=\sum_{k \in \mathbb{N}_{0}} \frac{F^{\prime \prime}(q)}{F^{\prime}\left(q\left(1-\beta^{k}\right)\right)} \beta^{k} .
$$

We see that last two series converge. Designating

$$
\frac{1}{A_{1}(s)}:=\frac{1}{q-s}+\frac{\Delta_{1}}{2} \quad \text { and } \quad \frac{1}{A_{2}(s)}:=\frac{1}{q-s}+\frac{\Delta_{2}}{2},
$$

we rewrite the relation (9) as following:

$$
\frac{1}{A_{1}(s)} \leq \lim _{n \rightarrow \infty} \frac{\beta^{n}}{R_{n}(s)} \leq \frac{1}{A_{2}(s)}
$$

Clearly that

$$
\frac{1}{A_{2}(s)}-\frac{1}{A_{1}(s)}=\frac{\Delta_{2}-\Delta_{1}}{2}<\infty .
$$

So there is a positive $\delta=\delta(s)$ such that $\Delta_{1} \leq \delta \leq \Delta_{2}$ and the limit in (10) is equal to

$$
\frac{1}{\mathscr{A}(s)}=\frac{1}{q-s}+\frac{\delta}{2}
$$

Having spent similar reasoning for $s \in[q ; 1)$ as before, we will be convinced that the $\operatorname{limit}_{n \rightarrow \infty} \lim ^{n} / R_{n}(s)=\mathscr{A}(s)$ holds for all $s \in[0 ; 1)$. 
So we can formulate the following basic lemma.

Lemma 1. The following assertions are true for all $s \in[0 ; 1)$ :

(i) if $A \neq 1$ and $F^{\prime \prime}(q)<\infty$, then

$$
R_{n}(s)=\mathscr{A}(s) \cdot \beta^{n}(1+o(1)) \text { as } n \rightarrow \infty,
$$

where the function $\mathscr{A}(s)$ is defined in (11);

(ii) (see [1,p.19]) if $A=1$ and $2 B:=F^{\prime \prime}(1)<\infty$, then

$$
R_{n}(s)=\frac{1-s}{(1-s) B n+1}(1+o(1)), \quad \text { as } n \rightarrow \infty
$$

The following lemma is discrete-time analogue of Lemma 2 from [5].

Lemma 2. The following assertions hold for all $s \in[0 ; 1)$ :

(i) if $A \neq 1$ and $F^{\prime \prime}(q)<\infty$, then

$$
R_{n}^{\prime}(s)=-\mathscr{K}(s) \cdot \beta^{n}(1+o(1)), \quad \text { as } n \rightarrow \infty,
$$

where $\mathscr{K}(s)=\exp \{-\delta \cdot \mathscr{A}(s)\}$ and $\delta=\delta(s) \in\left[\Delta_{1} ; \Delta_{2}\right]$;

(ii) if $A=1$ and $2 B:=F^{\prime \prime}(1)<\infty$, then

$$
R_{n}^{\prime}(s)=\frac{\hbar(s) B}{s-F(s)} R_{n}^{2}(s)(1+o(1)), \quad \text { as } n \rightarrow \infty,
$$

where $F^{\prime}(s) \leq \hbar(s) \leq 1$ and $R_{n}(s)$ has the expression (2.10).

Proof. Concerning the first part of the lemma we have equality

$$
\frac{R_{n+1}^{\prime}(s)}{R_{n}^{\prime}(s)}=\beta-F^{\prime \prime}\left(\xi_{n}(s)\right) R_{n}(s)
$$

Let at first $s \in[0 ; q)$. As the function $R_{n}(s)$ monotonously decreases by $s$, then its derivative $R_{n}^{\prime}(s)<0$ and, hence $R_{n+1}^{\prime}(s) / R_{n}^{\prime}(s)>0$. Therefore, taking the logarithm and after, summarizing along $n$, we transform the equality (16) to the form of

$$
\ln \left[-\frac{R_{n}^{\prime}(s)}{\beta^{n}}\right]=\sum_{k=0}^{n-1} \ln \left[1-\frac{F^{\prime \prime}\left(\xi_{k}(s)\right)}{\beta} R_{k}(s)\right]=: \sum_{k=0}^{n-1} \ln L_{k}(s)
$$

where

$$
L_{n}(s)=1-\frac{F^{\prime \prime}\left(\xi_{n}(s)\right)}{\beta} R_{n}(s)
$$

Using elementary inequalities

$$
\frac{b-a}{b}<\ln \frac{b}{a}<\frac{b-a}{a}, \text { where } 0<b<a,
$$

for $L_{k}(s)$ (a relevance of the use is easily be checked), we write

$$
\frac{L_{k}(s)-1}{L_{k}(s)}<\ln L_{k}(s)<L_{k}(s)-1 \text {. }
$$

In accordance with (5)

$$
-\frac{F^{\prime \prime}(q)}{\beta} R_{k}(s)<L_{k}(s)-1<-\frac{F^{\prime \prime}\left(q\left(1-\beta^{k}\right)\right)}{\beta} R_{k}(s)<0 .
$$


On the other hand as $R_{n}(s)<q \cdot \beta^{n}$, then $F_{n}(s)>q \cdot\left(1-\beta^{n}\right)$ and hence

$$
\beta L_{k}(s)=F^{\prime}\left(F_{k}(s)\right)>F^{\prime}\left(q\left(1-\beta^{k}\right)\right)
$$

Combining of relations (18)-(19) yields

$$
-\frac{F^{\prime \prime}(q)}{F^{\prime}\left(q\left(1-\beta^{k}\right)\right)} R_{k}(s)<\ln L_{k}(s)<-\frac{F^{\prime \prime}\left(q\left(1-\beta^{k}\right)\right)}{\beta} R_{k}(s) .
$$

Using this relation in (17) we obtain

$$
\sum_{k=0}^{n-1} \frac{F^{\prime \prime}\left(q\left(1-\beta^{k}\right)\right)}{\beta} R_{k}(s)<\ln \left[-\frac{\beta^{n}}{R_{n}^{\prime}(s)}\right]<\sum_{k=0}^{n-1} \frac{F^{\prime \prime}(q)}{F^{\prime}\left(q\left(1-\beta^{k}\right)\right)} R_{k}(s) .
$$

Hence in our designations

$$
A_{2}(s) \cdot \Delta_{1} \leq \lim _{n \rightarrow \infty} \ln \left[-\frac{\beta^{n}}{R_{n}^{\prime}(s)}\right] \leq A_{1}(s) \cdot \Delta_{2},
$$

Since $\Delta_{1} \leq \delta \leq \Delta_{2}$, owing to (10)-(12)

$$
A_{2}(s) \leq \lim _{n \rightarrow \infty} \frac{R_{n}(s)}{\beta^{n}}=\mathscr{A}(s) \leq A_{1}(s)
$$

Considering together the estimations (21) and (22) we conclude

$$
\Delta_{1} \leq \lim _{n \rightarrow \infty} \frac{\ln \left[-\frac{\beta^{n}}{R_{n}^{\prime}(s)}\right]}{\mathscr{A}(s)} \leq \Delta_{2} .
$$

The function $\beta^{n} / R_{n}^{\prime}(s)$ is continuous and monotone by $s$ for each $n \in \mathbb{N}_{0}$. Inequalities (23) entail that the functions $\ln \left[-\beta^{n} / R_{n}^{\prime}(s)\right]$ converge uniformly for $0 \leq s \leq z<q$ as $n \rightarrow \infty$. From here we get (14) for $0 \leq s<q$. By similar reasoning we will be convinced that convergence (14) is fair for $s \in[q ; 1)$ and ergo for all values of $s$, such that $0 \leq s<1$.

Let's prove now the formula (15). The Taylor expansion and iteration of $F(s)$ produce

$$
F_{n}(F(s))-F_{n}(s)=B R_{n}^{2}(s)(1+o(1)), \quad \text { as } n \rightarrow \infty .
$$

In the left-side part of (24) we apply the mean value Theorem and have

$$
F_{n}^{\prime}(c(s))=\frac{B}{F(s)-s} R_{n}^{2}(s)(1+o(1)), \quad \text { as } n \rightarrow \infty,
$$

where $s<c(s)<F(s)$. If we use a derivative's monotonicity property of any GF, a functional iteration of $F(s)$ entails

$$
F_{n}^{\prime}(s)<F_{n}^{\prime}(c(s))<\frac{F_{n+1}^{\prime}(s)}{F^{\prime}(s)} .
$$

From here, using iteration again we have

$$
\frac{F^{\prime}(s)}{F^{\prime}\left(F_{n}(s)\right)} F_{n}^{\prime}(c(s))<F_{n}^{\prime}(s)<F_{n}^{\prime}(c(s))
$$


It follows from relations (25), (26) and the fact $F_{n}(s) \uparrow 1$, that

$$
F^{\prime}(s) \leq \lim _{n \rightarrow \infty} \frac{(F(s)-s) F_{n}^{\prime}(s)}{B R_{n}^{2}(s)} \leq 1
$$

Designating $\hbar(s)$ the mid-part of last inequalities leads us to the representation (15). Lemma 2 is proved.

Remark. The function $\mathscr{A}(s)$ plays the same role, as the akin function in the Basic Lemma for the continuous-time Markov branching process established in [5]; see also [7]. Really, it can check up that in the conditions of the Lemma 1, $0<$ $\mathscr{A}(0)<\infty, \mathscr{A}(q)=0, \mathscr{A}^{\prime}(q)=-1$, and also it is asymptotically satisfied to the Schroeder equation:

$$
\mathscr{A}\left(F_{n}(q s)\right)=\beta^{n} \cdot \mathscr{A}(q s)(1+o(1)), \quad \text { as } n \rightarrow \infty,
$$

for all $0 \leq s<1$.

Now due to the Lemma 2 we can calculate the probability of return to an initial state $Z_{0}=1$ in time $n$. So since $F_{n}^{\prime}(0)=$ $P_{11}(n)$, putting $s=0$ in (14) and (15) we directly obtain the following two local limit theorems.

Theorem 1. Let $A \neq 1$ and $F^{\prime \prime}(q)<\infty$. Then

$$
\beta^{-n} P_{11}(n)=\mathscr{K}(0)(1+o(1)), \quad \text { as } n \rightarrow \infty,
$$

where the function $\mathscr{K}(s)$ is defined in (14).

Theorem 2. If $A=1$ and the second moment $F^{\prime \prime}(1)=: 2 B$ is finite, then

$$
n^{2} P_{11}(n)=\frac{\widehat{p}_{1}}{p_{0} B}(1+o(1)), \quad \text { as } n \rightarrow \infty
$$

whenever $p_{1} \leq \widehat{p}_{1} \leq 1$.

\section{An ergodic behavior of transition functions $\left\{P_{i j}(n)\right\}$ and invariant measures}

We devote this section to ergodicity property of transition functions $\left\{P_{i j}(n)\right\}$. Herewith we will essentially use the Lemma 2 with combining the following ratio limit property (RLP) [1].

Lemma 3. [see [1, p.15]] If $p_{1} \neq 0$, then for all $i, j \in \mathscr{S}$ the RLP holds:

$$
\frac{P_{i j}(n)}{P_{11}(n)} \longrightarrow i q^{i-1} \mu_{j}<\infty, \quad \text { as } n \rightarrow \infty,
$$

where $\mu_{j}=\lim _{n \rightarrow \infty} P_{1 j}(n) / P_{11}(n)$.

Denoting

$$
\mathscr{M}_{n}^{(i)}(s)=\sum_{j \in \mathscr{S}} \frac{P_{i j}(n)}{P_{11}(n)} s^{j}
$$

we see that a GF analogue of assertion (29) is

$$
\mathscr{M}_{n}^{(i)}(s) \sim i q^{i-1} \mathscr{M}_{n}(s) \longrightarrow i q^{i-1} \mathscr{M}(s)<\infty, \quad \text { as } n \rightarrow \infty,
$$

here $\mathscr{M}_{n}(s)=\mathscr{M}_{n}^{(1)}(s)$ and $\mathscr{M}(s)=\sum_{j \in \mathscr{S}} \mu_{j} s^{j}$. The properties of numbers $\left\{\mu_{j}\right\}$ are of some interest within our purpose. In view of their non-negativity the limiting GF $\mathscr{M}(s)$ is monotonously not decreasing by $s$. And according to 
the assertion (30) in studying of behavior of $P_{i j}(n) / P_{11}(n)$ is enough to consider function $\mathscr{M}_{n}(s)$.

It has been proved in [1, pp.12-14] the sequence $\left\{\mu_{j}\right\}$ satisfies to equation

$$
\beta \mu_{j}=\sum_{k \in \mathscr{S}} \mu_{k} P_{k j}, \quad \text { for all } j \in \mathscr{S}
$$

where $P_{i j}=\mathbb{P}_{i}\left\{Z_{1}=j\right\}$. Therewith the GF $\mathscr{M}(s)$ satisfies to the functional equation

$$
\mathscr{M}(F(s))=\beta \mathscr{M}(s)+\mathscr{M}\left(p_{0}\right)
$$

whenever $s$ and $p_{0}$ are in the region of convergence of $\mathscr{M}(s)$.

The following theorem describes main properties of this function.

Theorem 3. Let $p_{1} \neq 0$. Then $\mathscr{M}(s)$ converges for $0 \leq s<1$. Furthermore

(i) if $A \neq 1$ and $F^{\prime \prime}(q)<\infty$, then

$$
\mathscr{M}(s)=\frac{\mathscr{A}(0)-\mathscr{A}(s)}{\mathscr{K}(0)}
$$

whenever $\mathscr{A}(s)$ and $\mathscr{K}(s)$ are functions in (12) and (14) respectively;

(ii) if $A=1$ and $2 B:=F^{\prime \prime}(1)<\infty$, then $\mathscr{M}_{n}(s)=\mathscr{M}(s)+r_{n}(s)$, where

$$
\mathscr{M}(s)=\frac{p_{0}}{\widehat{p}_{1} B} \cdot \frac{s}{1-s}
$$

$$
\text { and } p_{1} \leq \widehat{p}_{1} \leq 1, r_{n}(s)=\mathscr{O}(1 / n) \text { as } n \rightarrow \infty
$$

Proof. The convergence property of GF $\mathscr{M}(s)$ was proved in [1, p.13].

In our designations we write

$$
\mathscr{M}_{n}(s)=\frac{F_{n}(s)-F_{n}(0)}{F_{n}^{\prime}(0)}=\left(1-\frac{R_{n}(s)}{R_{n}(0)}\right) \cdot \frac{R_{n}(0)}{P_{11}(n)} .
$$

In case $A \neq 1$ it follows from (12) that

$$
\frac{R_{n}(s)}{R_{n}(0)} \longrightarrow \frac{\mathscr{A}(s)}{\mathscr{A}(0)}, \text { as } n \rightarrow \infty
$$

and, considering (27) implies

$$
\frac{R_{n}(0)}{P_{11}(n)} \longrightarrow \frac{\mathscr{A}(0)}{\mathscr{K}(0)} .
$$

Combining (35) and (36) we obtain $\mathscr{M}(s)$ in form of (33).

Let's pass to the case $A=1$. Due to statement of (2.10) appears

$$
1-\frac{R_{n}(s)}{R_{n}(0)} \sim \frac{s}{(1-s) B n+1}, \text { as } n \rightarrow \infty .
$$

In turn according to (28)

$$
\frac{R_{n}(0)}{P_{11}(n)} \sim \frac{p_{0}}{\widehat{p}_{1}} n, \quad \text { as } n \rightarrow \infty
$$


Considering together relations (35), (37) and (38) we obtain

$$
\mathscr{M}_{n}(s) \sim \frac{p_{0}}{\widehat{p}_{1}} \frac{s n}{(1-s) B n+1}, \quad \text { as } n \rightarrow \infty .
$$

Taking limit from here we find the limiting GF in the form of (34). The proof is completed.

Remark. The theorem above is an enhanced form of Theorem 2 from [1, p.13] in sense that in our case we get the information on analytical form of limiting GF $\mathscr{M}(s)$.

The following assertions follow from the theorem proved above.

Corollary 1. Let $p_{1} \neq 0$. Then

(i) if $A \neq 1$ and $F^{\prime \prime}(q)<\infty$, then

$$
\mathscr{M}(q)=\sum_{j \in \mathscr{S}} \mu_{j} q^{j}=\frac{\mathscr{A}(0)}{\mathscr{K}(0)}<\infty
$$

(ii) if $A=1$ and $2 B:=F^{\prime \prime}(1)<\infty$, then

$$
\sum_{j=1}^{n} \mu_{j} \sim \frac{p_{0}}{\widehat{p}_{1} B} n, \quad \text { as } n \rightarrow \infty .
$$

Proof. The relation (39) follows from (33). In case $A=1$ as shown in (34)

$$
\mathscr{M}(s) \sim \frac{p_{0}}{\widehat{p}_{1} B} \cdot \frac{1}{1-s}, \quad \text { as } s \uparrow 1 .
$$

According to the Hardy-Littlewood Tauberian theorem the last relation entails (40).

Now from the Lemma 3 and Theorems 1 and 2 we get complete account about asymptotic behaviors of transition functions $P_{i j}(n)$. Following theorems are fair.

Theorem 4. Let $p_{1} \neq 0$. If $A \neq 1$ and $F^{\prime \prime}(q)<\infty$, then

$$
\beta^{-n} P_{i j}(n)=\frac{\mathscr{A}(0)}{\mathscr{M}(q)} i q^{i-1} \mu_{j}(1+o(1)), \quad \text { as } n \rightarrow \infty .
$$

Theorem 5. Let $p_{1} \neq 0$. If in critical GWP the second moment $F^{\prime \prime}(1)=: 2 B$ is finite then for transition functions the following asymptotic representation holds:

$$
n^{2} P_{i j}(n)=\frac{\widehat{p}_{1}}{p_{0} B} i \mu_{j}(1+o(1)), \quad \text { as } n \rightarrow \infty .
$$

Further we will discuss the role of the set $\left\{\mu_{j}\right\}$ as invariant measures concerning transition probabilities $\left\{P_{i j}(n)\right\}$. An invariant (or stationary) measure of the GWP is a set of nonnegative numbers $\left\{\mu_{j}^{*}\right\}$ satisfying to equation

$$
\mu_{j}^{*}=\sum_{k \in \mathscr{S}} \mu_{k}^{*} P_{k j}
$$

If $\sum_{j \in \mathscr{S}} \mu_{j}^{*}<\infty$ (or without loss of generality $\sum_{j \in \mathscr{S}} \mu_{j}^{*}=1$ ) then it is called as invariant distribution. As $P_{00}(n)=1$ then according to (41) $\mu_{0}^{*}=0$ for any invariant measure $\left\{\mu_{j}^{*}\right\}$. If $P_{10}(n)=0$ then condition (41) becomes $\mu_{j}^{*}=\sum_{k=1}^{j} \mu_{k}^{*} P_{k j}(n)$. If $P_{10}(n)>0$ then $P_{i 0}(n)>0$ and hence $\mu_{j}^{*}>0$. 
In virtue of Theorem 4 in non-critical situation the transition functions $P_{i j}(n)$ exponentially decrease to zero as $n \rightarrow \infty$. Following a classification of the continuous-time Markov process we characterize this decrease by a "decay parameter"

$$
\mathscr{R}=-\lim _{n \rightarrow \infty} \frac{\ln P_{i i}(n)}{n}
$$

We classify the non-critical Markov chain $\left\{Z_{n}, n \in \mathbb{N}_{0}\right\}$ as $\mathscr{R}$-transient if

$$
\sum_{n \in \mathbb{N}} e^{\mathscr{R} n} P_{i i}(n)<\infty
$$

and $\mathscr{R}$-recurrent otherwise. This chain is called as $\mathscr{R}$-positive if $\lim _{n \rightarrow \infty} e^{\mathscr{R} n} P_{i i}(n)>0$, and $\mathscr{R}$-null if last limit is equal to zero.

Now assertion(39) and Theorem 4 yield the following statement.

Theorem 6. Let $p_{1} \neq 0$. If $A \neq 1$ and $F^{\prime \prime}(q)<\infty$, then $\mathscr{R}=|\ln \beta|$ and the chain $\left\{Z_{n}\right\}$ is $\mathscr{R}$-positive. The set of numbers $\left\{\mu_{j}\right\}$ determined by GF (33) is the unique (up to multiplicative constant) $\mathscr{R}$-invariant measure for GWP.

In critical situation the set $\left\{\mu_{j}\right\}$ directly enters to a role of invariant measure for the GWP. Indeed, in this case $\beta=1$ and according to (31) the following invariant equation holds:

$$
\mu_{j}=\sum_{k \in \mathscr{S}} \mu_{k} P_{k j}, \quad \text { for all } j \in \mathscr{S}
$$

and owing to (40) $\sum_{j \in \mathscr{S}} \mu_{j}=\infty$.

Remark. As shown in Theorems 4 and 5 hit probabilities of GWP to any states through the long interval time depend on the initial state. That is ergodic property for $\left\{Z_{n}, n \in \mathbb{N}_{0}\right\}$ is not carried out.

Our further reasoning is connected with earlier introduced variable

$$
\mathscr{H}:=\min \left\{n \in \mathbb{N}: Z_{n}=0\right\}
$$

which denote the extinction time of GWP. Let as before

$$
\mathbb{P}_{i}^{\mathscr{H}(n)}\{*\}:=\mathbb{P}_{i}\{* \mid n<\mathscr{H}<\infty\}
$$

Put into consideration probabilities $\widetilde{P}_{i j}(n)=\mathbb{P}_{i}^{\mathscr{H}(n)}\left\{Z_{n}=j\right\}$ and denote

$$
\mathscr{V}_{n}^{(i)}(s)=\sum_{j \in \mathscr{S}} \widetilde{P}_{i j}(n) s^{j}
$$

to be the appropriate GF. As it has been noticed in the introduction section that if $q>0$, then the $\operatorname{limit} v_{j}:=\lim _{n \rightarrow \infty} \widetilde{P}_{1 j}(n)$ always exists. In case of $A \neq 1$ the set $\left\{v_{j}\right\}$ represents a probability distribution. And limiting GF $\mathscr{V}(s)=\sum_{j \in \mathscr{S}} v_{j} s^{j}$ satisfies to Schroeder's equation (1.3) for $0 \leq s \leq 1$. But if $A=1$ then $v_{j} \equiv 0$; see [19] and [1, p.16]. In forthcoming two theorems we observe the limit of $\widetilde{P}_{i j}(n)$ as $n \rightarrow \infty$ for any $i, j \in \mathscr{S}$. Unlike aforementioned results of Seneta we get the explicit expressions for the appropriate GF.

Theorem 7. Let $p_{1} \neq 0$. If $A \neq 1$ and $F^{\prime \prime}(q)<\infty$, then

$$
\lim _{n \rightarrow \infty} \widetilde{P}_{i j}(n)=v_{j}, \quad \text { for all } j \in \mathscr{S},
$$


and suitable $G F \mathscr{V}(s)=\sum_{j \in \mathscr{S}} v_{j} s^{j}$ has a form of

$$
\mathscr{V}(s)=1-\frac{\mathscr{A}(q s)}{\mathscr{A}(0)}
$$

where the function $\mathscr{A}(s)$ is defined in (11).

Proof. We write

$$
\widetilde{P}_{i j}(n)=\frac{\mathbb{P}_{i}\left\{Z_{n}=j, n<\mathscr{H}<\infty\right\}}{\mathbb{P}_{i}\{n<\mathscr{H}<\infty\}} .
$$

In turn

$$
\mathbb{P}_{i}\left\{Z_{n}=j, n<\mathscr{H}<\infty\right\}=\mathbb{P}\left\{n<\mathscr{H}<\infty \mid Z_{n}=j\right\} \cdot P_{i j}(n) .
$$

Since the vanishing probability of $j$ particles is equal to $q^{j}$ then from last form we receive that

$$
\mathbb{P}_{i}\left\{Z_{n}=j, n<\mathscr{H}<\infty\right\}=q^{j} \cdot P_{i j}(n)
$$

Using relation (44) implies

$$
\mathbb{P}_{i}\{n<\mathscr{H}<\infty\}=\sum_{j \in \mathscr{S}} \mathbb{P}_{i}\left\{Z_{n}=j, n<\mathscr{H}<\infty\right\}=\sum_{j \in \mathscr{S}} P_{i j}(n) q^{j}
$$

Now it follows from (43)-(45) and Lemma 3 that

$$
\widetilde{P}_{i j}(n)=\frac{\frac{P_{i j}(n)}{P_{11}(n)} \cdot q^{j}}{\sum_{k \in \mathscr{S}} \frac{P_{i k}(n)}{P_{11}(n)} q^{k}} \longrightarrow \frac{\mu_{j} \cdot q^{j}}{\sum_{k \in \mathscr{S}} \mu_{k} q^{k}}=\frac{\mu_{j} q^{j}}{\mathscr{M}(q)}=: v_{j}
$$

as $n \rightarrow \infty$. It can be verified the limit distribution $\left\{v_{j}\right\}$ defines the GF $\mathscr{V}(s)=\mathscr{M}(q s) / \mathscr{M}(q)$. Applying here equality (33) we get to (42).

Remark. The mean of distribution measure $\widetilde{P}_{i j}(n)$

$$
\sum_{j \in \mathscr{S}} j \widetilde{P}_{i j}(n) \longrightarrow \frac{q}{\mathscr{A}(0)}, \text { as } n \rightarrow \infty
$$

and, the limit distribution $\left\{v_{j}\right\}$ has the finite mean $\mathscr{V}^{\prime}(s \uparrow 1)=q / \mathscr{A}(0)$.

Further consider the case $A=1$. In this case $\mathbb{P}\{\mathscr{H}<\infty\}=1$, therefore

$$
\mathscr{V}_{n}^{(i)}(s)=\sum_{j \in \mathscr{S}} \mathbb{P}_{i}\left\{Z_{n}=j \mid \mathscr{H}>n\right\} s^{j}=\sum_{j \in \mathscr{S}} \frac{P_{i j}(n)}{\mathbb{P}_{i}\left\{Z_{n}>0\right\}} s^{j}=1-\frac{1-F_{n}^{i}(s)}{1-F_{n}^{i}(0)} .
$$

We see that $1-F_{n}^{i}(s) \sim i R_{n}(s)$ as $n \rightarrow \infty$. Hence considering (35) obtains

$$
\mathscr{V}_{n}^{(i)}(s) \sim 1-\frac{R_{n}(s)}{R_{n}(0)}=\frac{P_{11}(n)}{R_{n}(0)} \cdot \mathscr{M}_{n}(s), \quad \text { as } n \rightarrow \infty .
$$

Combining expansions (2.10), (28), (34) and (46), we state the following theorem. 
Theorem 8. Let $A=1$. If $2 B:=F^{\prime \prime}(1)<\infty$, then

$$
n \mathscr{V}_{n}^{(i)}(s)=\frac{1}{B} \cdot \frac{s}{1-s}+\rho_{n}(s)
$$

where $\rho_{n}(s)=\mathscr{O}(1 / n)$ as $n \rightarrow \infty$.

Remark. It is a curious fact that in last theorem we managed to be saved of undefined variable $\widehat{p}_{1} \in\left[p_{1} ; 1\right]$.

Now define the stochastic process $\widetilde{Z}_{n}$ with the transition matrix $\left\{\widetilde{P}_{i j}(n)\right\}$. It is easy to be convinced that $\widetilde{Z}_{n}$ represents a discrete-time Markov chain. According to last theorems the properties of its trajectory lose independence on initial state with growth the numbers of generations.

In non-critical case, according to the Theorem 7, for GWP $\widetilde{Z}_{n}$ there is (up to multiplicative constant) unique set of nonnegative numbers $\left\{v_{j}\right\}$ which are not all zero and $\sum_{j \in \mathscr{S}} v_{j}=1$. Moreover as $\mathscr{M}(q s)=\mathscr{M}(q) \cdot \mathscr{V}(s)$ then using the formula (32) we can establish the following invariant equation:

$$
\beta \cdot \mathscr{V}(s)=\mathscr{V}(\widehat{F}(s))-\mathscr{V}(\widehat{F}(s))
$$

where $\mathscr{V}(s)=\sum_{j \in \mathscr{S}} v_{j} s^{j}$ and $\widehat{F}(s)=F(q s) / q$.

So we have the following

Theorem 9. Let $A \neq 1$ and $F^{\prime \prime}(q)<\infty$. Then

$$
P_{i j}(n)=\widetilde{P}_{i j}(n) \cdot \sum_{k \in \mathscr{S}} P_{i k}(n) q^{k-j}
$$

where transition functions $\widetilde{P}_{i j}(n)$ have an ergodic property and their limits $v_{j}=\lim _{n \rightarrow \infty} \widetilde{P}_{i j}(n)$ present $|\ln \beta|$-invariant distribution for the Markov chain $\left\{\widetilde{Z}_{n}\right\}$.

In critical situation we have the following assertion which directly implies from Theorem 8 and taking into account the continuity theorem for GF.

Theorem 10. If in critical GWP $2 B:=F^{\prime \prime}(1)<\infty$, then

$$
n \widetilde{P}_{i j}(n)=\frac{1}{B}+\mathscr{O}\left(\frac{1}{n}\right), \quad \text { as } n \rightarrow \infty .
$$

\section{Limiting interpretation of $\mathbb{P}_{i}^{\mathscr{H}(n+k)}\{*\}$}

In this section, excepting cases $p_{1}=0$ and $q=0$, we observe the distribution $\mathbb{P}_{i}^{\mathscr{H}(n+k)}\left\{Z_{n}=j\right\}$. It has still been noticed by Harris [4] that its limit as $k \rightarrow \infty$ always exists for any fixed $n \in \mathbb{N}$. By means of relations (43)-(45) it was obtained in $[1, \mathrm{pp} .56-60]$ that

$$
\lim _{k \rightarrow \infty} \mathbb{P}_{i}^{\mathscr{H}(n+k)}\left\{Z_{n}=j\right\}=\frac{j q^{j-i}}{i \beta^{n}} P_{i j}(n)=: \mathscr{Q}_{i j}(n) .
$$

Since $F_{n}^{\prime}(q)=\left[F^{\prime}(q)\right]^{n}=\beta^{n}$, then by (1.2)

$$
\sum_{j \in \mathscr{S}} \frac{j q^{j-i}}{i \beta^{n}} P_{i j}(n)=\frac{1}{i q^{i-1} \beta^{n}}\left[\sum_{j \in \mathscr{S}} P_{i j}(n) s^{j}\right]_{s=q}^{\prime}=1 .
$$


So we have an honest probability measure $\mathbf{Q}=\left\{\mathscr{Q}_{i j}(n)\right\}$. The stochastic process $\left\{W_{n}, n \in \mathbb{N}_{0}\right\}$ defined by this measure is called the Q-process.

By definition

$$
\mathbf{Q}=\left\{\lim _{k \rightarrow \infty} \mathbb{P}_{i}\{* \mid n+k<\mathscr{H}<\infty\}\right\}=\left\{\mathbb{P}_{i}\{* \mid \mathscr{H}=\infty\}\right\},
$$

that the Q-process can be considered as GWP with a non-degenerating trajectory in remote future, that is it conditioned on event $\{\mathscr{H}=\infty\}$. Harris [4] has established that if $A=1$ and $2 B:=F^{\prime \prime}(1)<\infty$ the distribution of $Z_{n} / B n$ conditioned on $\{\mathscr{H}=\infty\}$ has the limiting Erlang's law. Thus the Q-process $\left\{W_{n}, n \in \mathbb{N}_{0}\right\}$ represents a homogeneous Markov chain with initial state $W_{0} \stackrel{d}{=} Z_{0}$ and general state space which will henceforth denoted as $\mathscr{E} \subset \mathbb{N}$. The variable $W_{n}$ denote the state size of this chain in instant $n$ with the transition matrix

$$
\mathscr{Q}_{i j}(n)=\mathbb{P}_{i}\left\{W_{n+k}=j\right\}=\frac{j q^{j-i}}{i \beta^{n}} P_{i j}(n), \quad \text { for all } i, j \in \mathscr{E}, \quad \text { and for any } n, k \in \mathbb{N},
$$

and for any $n, k \in \mathbb{N}$.

Put into consideration a GF

$$
Y_{n}^{(i)}(s):=\sum_{j \in \mathscr{E}} \mathscr{Q}_{i j}(n) s^{j}
$$

From (1.2) and (47) we have

$$
Y_{n}^{(i)}(s)=\sum_{j \in \mathscr{E}} \frac{j q^{j-i}}{i \beta^{n}} P_{i j}(n) s^{j}=\frac{q^{1-i} s}{i \beta^{n}} \sum_{j \in \mathscr{E}} P_{i j}(n)(q s)^{j-1}=\frac{q s}{i \beta^{n}} \frac{\partial}{\partial x}\left[\left(\frac{F_{n}(x)}{q}\right)^{i}\right]_{x=q s} .
$$

Therefore

$$
Y_{n}^{(i)}(s)=\left[\frac{F_{n}(q s)}{q}\right]^{i-1} Y_{n}(s)
$$

where GF $Y_{n}(s):=Y_{n}^{(1)}(s)=\mathbb{E}\left[s^{W_{n}} \mid W_{0}=1\right]$ has the form of

$$
Y_{n}(s)=s \frac{F_{n}^{\prime}(q s)}{\beta^{n}}, \text { for all } n \in \mathbb{N}
$$

As $F_{n}(s) \rightarrow q$ owing to (48) and (49), $\mathscr{Q}_{i j}(n) / \mathscr{Q}_{1 j}(n) \rightarrow 1$, at infinite growth of the number of generations. Using (48) and iterating $F(s)$ produce a following functional relation:

$$
Y_{n+1}^{(i)}(s)=\frac{Y(s)}{\widehat{F}(s)} Y_{n}^{(i)}(\widehat{F}(s)),
$$

where $\widehat{F}(s)=F(q s) / q$ and $Y(s):=Y_{1}(s)$. We see that Q-process is completely defined by GF

$$
Y(s)=s \frac{F^{\prime}(q s)}{\beta}
$$

and, its evolution is regulated by the positive parameter $\beta$. In fact, if the first moment $\alpha:=Y^{\prime}(1)$ is finite then differentiating of (49) in $s=1$ gives

$$
\mathbb{E}_{i} W_{n}=(i-1) \beta^{n}+\mathbb{E} W_{n}
$$


and

$$
\mathbb{E} W_{n}=\left\{\begin{array}{l}
1+\gamma\left(1-\beta^{n}\right), \quad \text { when } \beta<1, \\
(\alpha-1) n+1, \quad \text { when } \beta=1,
\end{array}\right.
$$

where $\gamma:=(\alpha-1) /(1-\beta)$ and $\alpha=1+\widehat{F}^{\prime \prime}(1) / \beta>1$.

\section{Classification and ergodic behavior of states of Q-processes}

The formula (51) shows that if $\beta<1$, then

$$
\mathbb{E}_{i} W_{n} \longrightarrow 1+\gamma, \text { as } n \rightarrow \infty
$$

and, provided that $\beta=1$

$$
\mathbb{E}_{i} W_{n} \sim(\alpha-1) n, \text { as } n \rightarrow \infty .
$$

The Q-Process has the following properties:

(i) if $\beta<1$, then it is positive-recurrent;

(ii) if $\beta=1$, then it is transient.

In the transient case $W_{n} \rightarrow \infty$ with probability 1 ; see [1, p.59].

Let's consider first the positive-recurrent case. In this case according to (14), (48), (49) the limit $\pi(s):=\lim _{n \rightarrow \infty} Y_{n}^{(i)}(s)$ exists provided that $\alpha<\infty$. Then owing to (50) we make sure that GF $\pi(s)=\sum_{j \in \mathscr{E}} \pi_{j} s^{j}$ satisfies to invariant equation $\pi(s) \cdot F(q s) / q=Y(s) \cdot \pi(F(q s) / q)$. Applying this equation reduces to

$$
\pi(s)=\frac{Y_{n}(s)}{\widehat{F}_{n}(s)} \pi\left(\widehat{F}_{n}(s)\right)
$$

where $\widehat{F}_{n}(s)=F_{n}(q s) / q$. A transition function analogue of (52) is form of $\pi_{j}=\sum_{i \in \mathscr{E}} \pi_{i} \mathscr{Q}_{i j}(n)$. Taking limit in (52) as $n \rightarrow \infty$ it follows that $\pi\left(\widehat{F}_{n}(s)\right) \sim \widehat{F}_{n}(s)$ and it in turn entails $\sum_{j \in \mathscr{E}} \pi_{j}=1$ since $\widehat{F}_{n}(s) \rightarrow 1$. So in this case the set $\left\{\pi_{j}, j \in \mathscr{E}\right\}$ represents an invariant distribution. Differentiation (52) and taking into account (51) we easily compute that

$$
\pi^{\prime}(1)=\sum_{j \in \mathscr{E}} j \pi_{j}=1+\gamma
$$

where as before $\gamma:=(\alpha-1) /(1-\beta)$.

Further we note that owing to (14) and (48)

$$
\pi(s)=\operatorname{sexp}\{-\delta(q s) \cdot \mathscr{A}(q s)\}
$$

where the function $\mathscr{A}(s)$ looks like (11). Since $\pi(1)=1$ and $\mathscr{A}(q s)=\mathscr{O}(1-s)$ as $s \uparrow 1$ it is necessary to be

$$
\delta(q s)=\mathscr{O}\left((1-s)^{-\sigma}\right)
$$

with $\sigma<1$. On the other hand for feasibility of equality (53) is equivalent to that

$$
\left.\frac{\partial[\delta(q s) \cdot \mathscr{A}(q s)]}{\partial s}\right|_{s \uparrow 1}=-\gamma .
$$


If we remember the form of function $\mathscr{A}(s)$ the last condition becomes

$$
\lim _{s \uparrow 1}\left\{\delta^{\prime}(q s)\left[q(1-s)-\frac{\delta(q s)}{2} q^{2}(1-s)^{2}\right]-q \delta(q s)\right\}=-\gamma .
$$

For the function $\delta=\delta(s)$ all cases are disregarded except for the unique case $\sigma=0$ for the following simple reason. All functions having a form of $(1-s)^{-\sigma}$ monotonically increase to infinity as $s \uparrow 1$ when $0<\sigma<1$ and this fact contradicts the boundedness of function $\delta=\delta(s)$. In the case $\sigma<0$ cannot be occurred (54) since the limit in the left-hand part is equal to zero while $\gamma \neq 0$. In unique case $\sigma=0$ the limit is constant and in view of (54)

$$
\delta=\frac{\gamma}{q}
$$

We proved the following theorem.

Theorem 11. If $\beta<1$ and $\alpha:=Y^{\prime}(1)<\infty$, then for $0 \leq s<1$

$$
\lim _{n \rightarrow \infty} Y_{n}^{(i)}(s)=\pi(s)
$$

where $\pi(s)$ is probability GF having a form of

$$
\pi(s)=s \exp \left\{-\frac{\gamma(1-s)}{1+\frac{\gamma}{2}(1-s)}\right\} .
$$

The set $\left\{\pi_{j}, j \in \mathscr{E}\right\}$ coefficients in power series expansion of $\pi(s)=\sum_{j \in \mathscr{E}} \pi_{j} s^{j}$ are invariant distribution for the Q-process.

In transient case the following theorem hold.

Theorem 12. If $\beta=1$ and $\alpha:=Y^{\prime}(1)<\infty$, then for all $0 \leq s<1$

$$
n^{2} Y_{n}^{(i)}(s)=\mu(s)\left(1+r_{n}(s)\right), \quad \text { as } n \rightarrow \infty,
$$

where $r_{n}(s)=o(1)$ for $0 \leq s<1$ and the $G F \mu(s)=\sum_{j \in \mathscr{E}} \mu_{j} s^{j}$ has a form of

$$
\mu(s)=\frac{2 s \hbar(s)}{(\alpha-1)(F(s)-s)}
$$

with $Y(s) \leq s \hbar(s) \leq s$. Nonnegative numbers $\left\{\mu_{j}, j \in \mathscr{E}\right\}$ satisfy to invariant equation

$$
\mu_{j}=\sum_{i \in \mathscr{E}} \mu_{i} \mathscr{Q}_{i j}(n)
$$

Moreover $\sum_{j \in \mathscr{E}} \mu_{j}=\infty$.

Proof. The convergence (56) immediately follows as a result of combination of (15), (48) and (49). Taking limit in (50) reduces to equation $\mu(s) F_{n}(s)=Y_{n}(s) \mu\left(F_{n}(s)\right)$ which equivalent to (57) in the context of transition probabilities. On the other hand it follows from (56) that $\mu\left(F_{n}(s)\right) \sim n^{2} F_{n}(s)$ as $n \rightarrow \infty$. Hence $\sum_{j \in \mathscr{E}} \mu_{j}=\infty$.

As $\lim _{s \downarrow 0}\left[Y_{n}^{(i)}(s) / s\right]=\mathscr{Q}_{i 1}(n)$, the following two theorems imply from (55) and (56).

Corollary 2. If $\beta<1$ and $\alpha:=Y^{\prime}(1)<\infty$, then

$$
\mathscr{Q}_{i 1}(n)=e^{-2 \gamma /(2+\gamma)}(1+o(1)), \quad \text { as } n \rightarrow \infty .
$$


Corollary 3. If $\beta=1$ and $\alpha:=Y^{\prime}(1)<\infty$, then

$$
n^{2} \mathscr{Q}_{i 1}(n)=\frac{2 \widetilde{\mathscr{Q}}_{1}}{(\alpha-1) p_{0}}(1+o(1)), \quad \text { as } n \rightarrow \infty,
$$

here $\mathscr{Q}_{11}(1) \leq \widetilde{\mathscr{Q}}_{1} \leq 1$.

Theorem 13. Let $\beta=1$ and $\alpha:=Y^{\prime}(1)<\infty$. Then

$$
\lim _{n \rightarrow \infty} \frac{1}{n^{2}}\left[\mu_{1}+\mu_{2}+\cdots+\mu_{n}\right]=\frac{2}{(\alpha-1)^{2}} .
$$

Proof. By Taylor formula $F(s)-s \sim B(1-s)^{2}$ as $s \uparrow 1$. Therefore since $\lim _{s \uparrow 1} \hbar(s)=1$ for GF $\mu(s)$ we have

$$
\mu(s) \sim \frac{4}{(\alpha-1)^{2}} \frac{1}{(1-s)^{2}}, \quad \text { as } s \uparrow 1 .
$$

According to Hardy-Littlewood Tauberian theorem each of relations (60) and (61) entails another.

Another invariant measure for Q-process are numbers

$$
v_{j}:=\lim _{n \rightarrow \infty} \frac{\mathscr{Q}_{i j}(n)}{\mathscr{Q}_{i 1}(n)},
$$

which don't depend on $i \in \mathscr{E}$. In fact a similar way as in GWP (see Lemma 3) case it is easy to see that this limit exists. Owing to Kolmogorov-Chapman equation

$$
\frac{\mathscr{Q}_{i j}(n+1)}{\mathscr{Q}_{i 1}(n+1)} \frac{\mathscr{Q}_{i 1}(n+1)}{\mathscr{Q}_{i 1}(n)}=\sum_{k \in \mathscr{E}} \frac{\mathscr{Q}_{i k}(n)}{\mathscr{Q}_{i 1}(n)} \mathscr{Q}_{k j}(1)
$$

Last equality and (62), taking into account that $\mathscr{Q}_{i 1}(n+1) / \mathscr{Q}_{i 1}(n) \rightarrow 1$ gives us an invariant relation

$$
v_{j}=\sum_{i \in \mathscr{E}} v_{i} \mathscr{Q}_{i j}(1)
$$

In GF context the equality (63) is equivalent to Schroeder type functional equation

$$
\mathscr{U}(\widehat{F}(s))=\frac{\widehat{F}(s)}{Y(s)} \mathscr{U}(s),
$$

where $\widehat{F}_{n}(s)=F_{n}(q s) / q$ and

$$
\mathscr{U}(s)=\sum_{j \in \mathscr{E}} v_{j} s^{j}
$$

with $v_{1}=1$.

Note that in conditions of Theorem 11

$$
\mathscr{U}(s)=\pi(s) e^{2 \gamma /(2+\gamma)}
$$

Hence, considering (62), we generalize the statement (58):

$$
\mathscr{Q}_{i j}(n) \longrightarrow \pi_{j}=v_{j} e^{-2 \gamma /(2+\gamma)}, \quad \text { as } n \rightarrow \infty,
$$

for all $i, j \in \mathscr{E}$. 
By similar way for $\beta=1$ it is discovered that

$$
n^{2} \mathscr{Q}_{i j}(n) \longrightarrow \mu_{j}=v_{j} \frac{2 \widetilde{\mathscr{Q}}_{1}}{(\alpha-1) p_{0}}, \quad \text { as } n \rightarrow \infty
$$

where $\widetilde{\mathscr{Q}}_{1}$ is defined in (59).

Providing that $Y^{\prime \prime}(1)<\infty$ it can be estimated the convergence speed in Theorem 12. It is proved in [16] that if $C:=F^{\prime \prime \prime}(1)<\infty$, then

$$
R_{n}(s)=\frac{1}{b_{n}(s)}+\Delta \cdot \frac{\ln b_{n}(s)+K(s)}{\left(b_{n}(s)\right)^{2}}(1+o(1))
$$

as $n \rightarrow \infty$, where

$$
b_{n}(s)=\frac{F^{\prime \prime}(1)}{2} n+\frac{1}{1-s} \quad \text { and } \quad \Delta=\frac{C}{3 F^{\prime \prime}(1)}-\frac{F^{\prime \prime}(1)}{2},
$$

and $K(s)$ is some bounded function depending on form of $F(s)$. Since the finiteness of $C$ is equivalent to condition $Y^{\prime \prime}(1)<\infty$ then from combination of relations (15), (48), (49) and (64) we receive the following theorem for the case $\beta=1$.

Theorem 14. If together with conditions of Theorem 12 we suppose that $Y^{\prime \prime}(1)<\infty$, then for the error term in asymptotic formula (56) the following estimation holds:

$$
r_{n}(s)=\widetilde{\Delta} \cdot \frac{\ln b_{n}(s)}{b_{n}(s)}(1+o(1)), \quad \text { as } n \rightarrow \infty
$$

where $\widetilde{\Delta}$ is constant depending on the moment $Y^{\prime \prime}(1)$ and

$$
b_{n}(s)=\frac{(\alpha-1) n}{2}+\frac{1}{1-s} .
$$

Corollary 4. In conditions of Theorem 14 the following representation holds:

$$
n^{2} \mathscr{Q}_{i j}(n)=\mu_{j}\left(1+\frac{\Delta}{\alpha-1} \cdot \frac{\ln n}{n}(1+o(1))\right), \text { as } n \rightarrow \infty .
$$

\section{Joint distribution law of Q-process and its total state}

Consider the Q-process $\left\{W_{n}, n \in \mathbb{N}_{0}\right\}$ with structural parameter $\beta=F^{\prime}(q)$. Let's define a random variable

$$
S_{n}=W_{0}+W_{1}+\cdots+W_{n-1},
$$

a total state in Q-process until time $n$. Let

$$
J_{n}(s ; x)=\sum_{j \in \mathscr{E}} \sum_{l \in \mathbb{N}} \mathbb{P}\left\{W_{n}=j, S_{n}=l\right\} s^{j} x^{l}
$$

be the joint GF of $W_{n}$ and $S_{n}$ on a set of

$$
\mathbb{K}=\left\{(s ; x) \in \mathbb{R}^{2}:|s| \leq 1,|x| \leq 1, \sqrt{(s-1)^{2}+(x-1)^{2}} \geq r>0\right\} .
$$


Lemma 4. For all $(s ; x) \in \mathbb{K}$ and any $n \in \mathbb{N}$ a recursive equation

$$
J_{n+1}(s ; x)=\frac{Y(s)}{\widehat{F}(s)} J_{n}(x \widehat{F}(s) ; x)
$$

holds, where $Y(s)=s F^{\prime}(q s) / \beta$ and $\widehat{F}(s)=F(q s) / q$.

Proof. Let's consider the cumulative process $\left\{W_{n}, S_{n}\right\}$ which is evidently a bivariate Markov chain with transition functions

$$
\mathbb{P}\left\{W_{n+1}=j, S_{n+1}=l \mid W_{n}=i, S_{n}=k\right\}=\mathbb{P}_{i}\left\{W_{1}=j, S_{1}=l\right\} \delta_{l, i+k},
$$

where $\delta_{i j}$ is the Kronecker's delta function. Hence we have

$$
\begin{aligned}
\mathbb{E}_{i}\left[s^{W_{n+1}} x^{S_{n+1}} \mid S_{n}=k\right] & =\sum_{j \in \mathscr{E}} \sum_{l \in \mathbb{N}} \mathbb{P}_{i}\left\{W_{1}=j, S_{1}=l\right\} \delta_{l, i+k} s^{j} x^{l} \\
& =\sum_{j \in \mathscr{E}} \mathbb{P}_{i}\left\{W_{1}=j\right\} s^{j} x^{i+k}=Y^{(i)}(s) \cdot x^{i+k}
\end{aligned}
$$

Using this result and the formula of composite probabilities, we discover that

$$
\begin{aligned}
J_{n+1}(s ; x) & =\mathbb{E}\left[\mathbb{E}\left[s^{W_{n+1}} x^{S_{n+1}} \mid W_{n}, S_{n}\right]\right]=\mathbb{E}\left[Y^{\left(W_{n}\right)}(s) \cdot x^{W_{n}+S_{n}}\right] \\
& =\mathbb{E}\left[(\widehat{F}(s))^{W_{n}-1} \cdot Y(s) \cdot x^{W_{n}+S_{n}}\right] \\
& =\frac{Y(s)}{\widehat{F}(s)} \cdot \mathbb{E}\left[(x \widehat{F}(s))^{W_{n}} \cdot x^{S_{n}}\right] .
\end{aligned}
$$

The formula (4.2.) is used in last step. The last equation reduces to (65).

Now by means of relation (65) we can take an explicit expression for GF $J_{n}(s ; x)$. In fact, sequentially having applied it, taking into account(50) and, after some transformations we have

$$
J_{n}(s ; x)=s \prod_{k=0}^{n-1}\left[\frac{x \widehat{F}^{\prime}\left(H_{k}(s ; x)\right)}{\beta}\right]=\frac{s}{\beta^{n}} \frac{\partial H_{n}(s ; x)}{\partial s},
$$

where the sequence of functions $\left\{H_{k}(s ; x)\right\}$ is defined for $(s ; x) \in \mathbb{K}$ by following recurrence relations:

$$
\begin{aligned}
H_{0}(s ; x) & =s \\
H_{n+1}(s ; x) & =x \widehat{F}\left(H_{n}(s ; x)\right) .
\end{aligned}
$$

Since

$$
\left.\frac{\partial J_{n}(s ; x)}{\partial x}\right|_{(s ; x)=(1 ; 1)}=\mathbb{E} S_{n}
$$

then provided that $\alpha:=Y^{\prime}(1)$ it follows from 6.2) and (67) that

$$
\mathbb{E} S_{n}= \begin{cases}(1+\gamma) n-\gamma \frac{1-\beta^{n}}{1-\beta}, & \text { when } \beta<1, \\ \frac{\alpha-1}{2} n(n-1)+n, & \text { when } \beta=1,\end{cases}
$$

where as before $\gamma:=(\alpha-1) /(1-\beta)$. 
Remark. It is known from classical theory that if an evolution law of simple GWP $\left\{\widehat{Z_{n}}, n \in \mathbb{N}_{0}\right\}$ is generated by GF $\widehat{F}(s)=F(q s) / q$, then a joint GF of distribution of $\left\{\widehat{Z}_{n}, V_{n}\right\}$, where $V_{n}=\sum_{k=0}^{n-1} \widehat{Z_{k}}$ is the total number of individuals participating until time $n$, satisfies to the recurrent equation (67); see e.g., [14, p.126]. So $H_{n}(s ; x),(s ; x) \in \mathbb{K}$, represents the two-dimensional GF for all $n \in \mathbb{N}$ and has all properties as $\mathbb{E}\left[s^{\widehat{Z}_{n}} x^{V_{n}}\right]$.

In virtue of the told in Remark 6, in studying of function $H_{k}(s ; x)$ we certainly will use properties of GF $\mathbb{E}\left[s^{\widehat{Z}_{n}} x^{V_{n}}\right]$. As well as $\widehat{F}^{\prime}(1)=\beta \leq 1$ and hence the process $\left\{\widehat{Z}_{n}, n \in \mathbb{N}_{0}\right\}$ is mortal GWP. So there is an integer valued random variable $V=\lim _{n \rightarrow \infty} V_{n}$ - a total number of individuals participating in the process for all time of its evolution. Hence there is a limit

$$
h(x):=\mathbb{E} x^{V}=\lim _{n \rightarrow \infty} \mathbb{E} x^{V_{n}}=\lim _{n \rightarrow \infty} H_{n}(1 ; x)
$$

and according to (67) it satisfied the recurrence relation

$$
h(x)=x \widehat{F}(h(x)) .
$$

Provided that the second moment $Y^{\prime \prime}(1)$ is finite, the following asymptotes for the variances can be found from (66) by differentiation:

$$
\operatorname{Var} W_{n} \sim \begin{cases}\mathscr{O}(1), & \text { when } \beta<1, \\ \frac{(\alpha-1)^{2}}{2} n^{2}, & \text { when } \beta=1,\end{cases}
$$

and

$$
\operatorname{Var} S_{n} \sim \begin{cases}\mathscr{O}(n), & \text { when } \beta<1, \\ \frac{(\alpha-1)^{2}}{12} n^{4}, & \text { when } \beta=1,\end{cases}
$$

as $n \rightarrow \infty$. In turn it is matter of computation to verify that

$$
\operatorname{cov}\left(W_{n}, S_{n}\right) \sim \begin{cases}\mathscr{O}(1), & \text { when } \beta<1, \\ \frac{(\alpha-1)^{2}}{6} n^{3}, & \text { when } \beta=1 .\end{cases}
$$

Hence letting $\rho_{n}$ denote the correlation coefficient of $W_{n}$ and $S_{n}$, we have

$$
\lim _{n \rightarrow \infty} \rho_{n}= \begin{cases}0, & \text { when } \beta<1, \\ \frac{\sqrt{6}}{3}, & \text { when } \beta=1 .\end{cases}
$$

Last statement specifies that in the case $\beta<1$ between the variables $W_{n}$ and $S_{n}$ there is an asymptotic independence property. Contrariwise for the case $\beta=1$ the following "joint theorem" holds, which has been proved in the paper [6].

Theorem 15. Let $\beta=1$ and $\alpha=Y^{\prime}(1)<\infty$. Then the two-dimensional process

$$
\left(\frac{W_{n}}{\mathbb{E} W_{n}} ; \frac{S_{n}}{\mathbb{E} S_{n}}\right)
$$


weakly converges to the two-dimensional random vector $(\boldsymbol{w} ; \mathbf{s})$ having the Laplace transform

$$
\mathbb{E}\left[e^{-\lambda \boldsymbol{w}-\theta \boldsymbol{s}}\right]=\left[\operatorname{ch} \sqrt{\theta}+\frac{\lambda}{2} \frac{\operatorname{sh} \sqrt{\theta}}{\sqrt{\theta}}\right]^{-2}, \lambda, \theta \in \mathbb{R}_{+}
$$

where chx $=\left(e^{x}+e^{-x}\right) / 2$ and $\operatorname{sh} x=\left(e^{x}-e^{-x}\right) / 2$.

Supposing $\lambda=0$ in Theorem 15 produces the following limit theorem for $S_{n}$.

Corollary 5. Let $\beta=1$ and $\alpha=Y^{\prime}(1)<\infty$. Then for $0<u<\infty$

$$
\lim _{n \rightarrow \infty} \mathbb{P}\left\{\frac{S_{n}}{\mathbb{E} S_{n}} \leq u\right\}=F(u)
$$

where the limit function $F(u)$ has the Laplace transform

$$
\int_{0}^{+\infty} e^{-\theta u} d F(u)=\operatorname{sech}^{2} \sqrt{\theta}, \quad \theta \in \mathbb{R}_{+} .
$$

Letting $\theta=0$ from the Theorem 15 we have the following assertion which was proved in the monograph [1, pp.59-60] with applying of the Helly's theorem.

Corollary 6. Let $\beta=1$ and $\alpha=Y^{\prime}(1)<\infty$. Then for $0<u<\infty$

$$
\lim _{n \rightarrow \infty} \mathbb{P}\left\{\frac{W_{n}}{\mathbb{E} W_{n}} \leq u\right\}=1-e^{-2 u}-2 u e^{-2 u} .
$$

Really, denoting $\psi_{n}(\lambda)=\Psi_{n}(\lambda ; 0)$ we have

$$
\psi_{n}(\lambda) \longrightarrow \frac{1}{\left[1+\frac{\lambda}{2}\right]^{2}}, \text { as } n \rightarrow \infty
$$

Here we have used that $\lim _{\theta \downarrow 0} \operatorname{sh} \sqrt{\theta} / \sqrt{\theta}=1$. The found Laplace transform corresponds to a distribution of the righthand side term in (70) produced as composition of two exponential laws with an identical density.

\section{Asymptotic properties of $S_{n}$ in case of $\beta<1$}

In this section we investigate asymptotic properties of distribution of $S_{n}$ in the case $\beta<1$. Consider the GF $T_{n}(x):=$ $\mathbb{E} x^{S_{n}}=J_{n}(1 ; x)$. Owing to (66) it has a form of

$$
T_{n}(x)=\prod_{k=0}^{n-1} u_{k}(x)
$$

where

$$
u_{n}(x)=\frac{x \widehat{F}^{\prime}\left(h_{n}(x)\right)}{\beta},
$$

and $\widehat{F}(s)=F(q s) / q, h_{n}(x)=\mathbb{E} x^{V_{n}}, V_{n}=\sum_{k=0}^{n-1} \widehat{Z_{k}}$.

In accordance with $(67) h_{n+1}(x)=x \widehat{F}\left(h_{n}(x)\right)$. Denoting

$$
R_{n}(x):=h(x)-h_{n}(x), n \in \mathbb{N}_{0}
$$


for $x \in \mathbb{K}$ we have

$$
R_{n}(x)=x\left[\widehat{F}(h(x))-\widehat{F}\left(h_{n-1}(x)\right)\right]=x \mathbb{E}\left[h(x)-h_{n-1}(x)\right]^{\widehat{Z}_{n}} \leq \beta R_{n-1}(x),
$$

since $|h(x)| \leq 1$ and $\left|h_{n}(s ; x)\right| \leq 1$. Therefore

$$
\left|R_{n}(x)\right| \leq \beta^{n-k}\left|R_{k}(x)\right|,
$$

for each $n \in \mathbb{N}$ and $k=0,1, \ldots, n$. Consecutive application of last inequality gives

$$
R_{n}(x)=\mathscr{O}\left(\beta^{n}\right) \longrightarrow 0,
$$

as $n \rightarrow \infty$ uniformly for $x \in \mathbb{K}$. Further, where the function $R_{n}(x)$ is used, we deal with set $\mathbb{K}$ in which this function certainly is not zero.

By Taylor expansion and taking into account (72), (69), we have

$$
R_{n+1}(x)=x \widehat{F}^{\prime}(h(x)) R_{n}(x)-x \frac{\widehat{F}^{\prime \prime}(h(x))+\eta_{n}(x)}{2} R_{n}^{2}(x),
$$

where $\left|\eta_{n}(x)\right| \rightarrow 0$ as $n \rightarrow \infty$ uniformly with respect to $x \in \mathbb{K}$. Since $R_{n}(x) \rightarrow 0$, formula (73) implies

$$
R_{n}(x)=\frac{R_{n+1}(x)}{x \widehat{F}^{\prime}(h(x))}(1+o(1))
$$

Owing to last equality we transform the formula (73) to a form of

$$
R_{n+1}(x)=x \widehat{F}^{\prime}(h(x)) R_{n}(x)-\left[\frac{\widehat{F}^{\prime \prime}(h(x))}{2 \widehat{F}^{\prime}(h(x))}+\varepsilon_{n}(x)\right] R_{n}(x) R_{n+1}(x)
$$

and, hence

$$
\frac{u(x)}{R_{n+1}(x)}=\frac{1}{R_{n}(x)}+v(x)+\varepsilon_{n}(x)
$$

where

$$
u(x)=x \widehat{F}^{\prime}(h(x)) \quad \text { and } \quad v(x)=\frac{\widehat{F}^{\prime \prime}(h(x))}{2 \widehat{F}^{\prime}(h(x))},
$$

and $\left|\varepsilon_{n}(x)\right| \leq \varepsilon_{n} \rightarrow 0$ as $n \rightarrow \infty$ for all $x \in \mathbb{K}$. Repeated use of (74) leads to the following representation for $R_{n}(x)$ :

$$
\frac{u^{n}(x)}{R_{n}(x)}=\frac{1}{h(x)-1}+\frac{v(x) \cdot\left[1-u^{n}(x)\right]}{1-u(x)}+\sum_{k=1}^{n} \varepsilon_{k}(x) u^{k}(x) .
$$

Note that the formula (75) was written out in monograph [14, p.130] for the critical case.

The expansions of functions $h(x)$ and $u(x)$ in neighborhood of $x=1$ will be useful for our further purpose.

Lemma 5. Let $\beta<1$. If $b:=\widehat{F}^{\prime \prime}(1)<\infty$, then for $h(x)=\mathbb{E} x^{V}$ the following relation holds:

$$
1-h(x) \sim \frac{1}{1-\beta}(1-x)-\frac{2 \beta(1-\beta)+b}{(1-\beta)^{3}}(1-x)^{2},
$$


as $x \uparrow 1$.

Proof. We write down the Taylor expansion as $x \uparrow 1$ :

$$
h(x)=1+h^{\prime}(1)(x-1)+h^{\prime \prime}(1)(x-1)^{2}+o(x-1)^{2} .
$$

In turn by direct differentiation from (69) we have

$$
h^{\prime}(x)=\frac{\widehat{F}(h(x))}{1-u(x)}
$$

and

$$
h^{\prime \prime}(x)=\frac{2 \widehat{F}^{\prime}(h(x)) h^{\prime}(x)+x \widehat{F}^{\prime \prime}(h(x))\left[h^{\prime}(x)\right]^{2}}{1-u(x)} .
$$

Letting $x \uparrow 1$ in last equalities entails $h^{\prime}(1)=1 /(1-\beta)$ and

$$
h^{\prime \prime}(1)=\frac{2 \beta(1-\beta)+b}{(1-\beta)^{3}}
$$

which together with (77) proves (76).

We remind that existence of the second moment $b:=\widehat{F}^{\prime \prime}(1)$ is equivalent to existence of $\alpha=Y^{\prime}(1)$ and $\gamma=b / \beta(1-\beta)$. We use it in the following assertion.

Lemma 6. Let $\beta<1$. If $b:=\widehat{F}^{\prime \prime}(1)<\infty$, then as $x \uparrow 1$ the following relation holds:

$$
u(x) \sim \beta x[1-\gamma(1-x)]+\frac{2 \beta(1-\beta)+b}{(1-\beta)^{3}} b x(1-x)^{2} .
$$

Proof. The relation (78) follows from Taylor power series expansion of function $\widehat{F}^{\prime}(h(x))$, taking into account therein Lemma 5.

The following Lemma 7 is a direct consequence of relation (76). And Lemma 8 implies from (78) and Lemma 7. Therein we consider the fact that $b=\beta(\alpha-1)$.

Lemma 7. Let $\beta<1$ and $\alpha<\infty$. Then as $\theta \rightarrow 0$

$$
h\left(e^{\theta}\right)-1 \sim \frac{1}{1-\beta} \theta+\frac{\beta(2+\gamma)}{(1-\beta)^{2}} \theta^{2} .
$$

Lemma 8. If $\beta<1$ and $\alpha<\infty$, then as $\theta \rightarrow 0$

$$
u\left(e^{\theta}\right) \sim \beta[1+(1+\gamma) \theta]+\beta \gamma \frac{1+\beta(1+\gamma)}{1-\beta} \theta^{2} .
$$

The following assertion hails from (75), (79) and (80).

Lemma 9. Let $\beta<1$ and $\alpha<\infty$. Then the following relation holds:

$$
\frac{R_{n}\left(e^{\theta}\right)}{u^{n}\left(e^{\theta}\right)} \sim \frac{1}{1-\beta} \theta+\frac{\beta(2+\gamma)}{(1-\beta)^{2}} \theta^{2}
$$

as $\theta \rightarrow 0$ and for each fixed $n \in \mathbb{N}$. 
Further the following lemma is required.

Lemma 10. Let $\beta<1$ and $\alpha<\infty$. Then the following relation holds:

$$
\ln \prod_{k=0}^{n-1} u_{k}\left(e^{\theta}\right) \sim-\left(1-\frac{u\left(e^{\theta}\right)}{\beta}\right) n-\frac{\beta \gamma(2+\gamma)}{1-\beta} \theta^{3} \sum_{k=0}^{n-1} u^{k}\left(e^{\theta}\right)
$$

as $\theta \rightarrow 0$ and for each fixed $n \in \mathbb{N}$.

Proof. Using inequalities $\ln (1-y) \geq-y-y^{2} /(1-y)$, which hold for $0 \leq y<1$, we have

$$
\ln \prod_{k=0}^{n-1} u_{k}\left(e^{\theta}\right)=\sum_{k=0}^{n-1} \ln \left\{1-\left[1-u_{k}\left(e^{\theta}\right)\right]\right\}=\sum_{k=0}^{n-1}\left[u_{k}\left(e^{\theta}\right)-1\right]+\rho_{n}^{(1)}(\theta)=: I_{n}(\theta)+\rho_{n}^{(1)}(\theta)
$$

where

$$
I_{n}(\theta)=-\sum_{k=0}^{n-1}\left[1-u_{k}\left(e^{\theta}\right)\right]
$$

and

$$
0 \geq \rho_{n}^{(1)}(\theta) \geq-\sum_{k=0}^{n-1} \frac{\left[1-u_{k}\left(e^{\theta}\right)\right]^{2}}{u_{k}\left(e^{\theta}\right)}
$$

It is easy to be convinced that the functional sequence $\left\{h_{k}(x)\right\}$ does not decrease on $k$. Then according to property of GF, the function $u_{k}\left(e^{\theta}\right)$ is also non-decreasing on $k$ for each fixed $n \in \mathbb{N}$ and $\theta \in \mathbb{R}$. Hence,

$$
0 \geq \rho_{n}^{(1)}(\theta) \geq \frac{1-u_{0}\left(e^{\theta}\right)}{u_{0}\left(e^{\theta}\right)} I_{n}(\theta)
$$

We can verify also that $1-u_{0}\left(e^{\theta}\right) \rightarrow 0$ as $\theta \rightarrow 0$. Then in accordance with (85) the second expression in (83) $\rho_{n}^{(1)}(\theta) \rightarrow 0$ provided that $I_{n}(\theta)$ has a finite limit as $\theta \rightarrow 0$.

Further, by Taylor expansion we have

$$
\widehat{F}^{\prime}(t)=\widehat{F}^{\prime}\left(t_{0}\right)-\widehat{F}^{\prime \prime}\left(t_{0}\right)\left(t_{0}-t\right)+\left(t_{0}-t\right) g\left(t_{0} ; t\right)
$$

where $g\left(t_{0} ; t\right)=\left(t_{0}-t\right) \widehat{F}^{\prime \prime \prime}(\tau) / 2$ and $t_{0}<\tau<t$. Using this expansion we write

$$
u_{k}(x)=\frac{u(x)}{\beta}-\frac{x \widehat{F}^{\prime \prime}(h(x))}{\beta} R_{k}(x)+R_{k}(x) g_{k}(x)
$$

herein $g_{k}(x)=x R_{k}(x) \widehat{F}^{\prime \prime \prime} / 2 \beta$ and $h_{k}(x)<\tau<h(x)$. Therefore

$$
u_{k}\left(e^{\theta}\right)=\frac{u\left(e^{\theta}\right)}{\beta}-\frac{e^{\theta} \widehat{F}^{\prime \prime}\left(h\left(e^{\theta}\right)\right)}{\beta} R_{k}\left(e^{\theta}\right)+R_{k}\left(e^{\theta}\right) g_{k}\left(e^{\theta}\right) .
$$

It follows from (84) and (86) that

$$
I_{n}(\theta)=-\left[1-\frac{u\left(e^{\theta}\right)}{\beta}\right] n-\frac{e^{\theta} \widehat{F}^{\prime \prime}\left(h\left(e^{\theta}\right)\right)}{\beta} \sum_{k=0}^{n-1} R_{k}\left(e^{\theta}\right)+\rho_{n}^{(2)}(\theta),
$$


where

$$
0 \leq \rho_{n}^{(2)}(\theta) \leq R_{0}\left(e^{\theta}\right) \sum_{k=0}^{n-1} g_{k}\left(e^{\theta}\right)
$$

In last estimation we used the earlier known inequality $\left|R_{n}(x)\right| \leq \beta^{n}\left|R_{0}(x)\right|$. Owingto the relation $(79) R_{0}\left(e^{\theta}\right)=\mathscr{O}(\theta)$ as $\theta \rightarrow 0$. In turn according to (72) $g_{k}\left(e^{\theta}\right)=\mathscr{O}\left(\beta^{k}\right) \rightarrow 0$ as $k \rightarrow \infty$ for all $\theta \in \mathbb{R}$. Hence,

$$
R_{0}\left(e^{\theta}\right) \sum_{k=0}^{n-1} g_{k}\left(e^{\theta}\right)=\mathscr{O}(\theta) \longrightarrow 0, \quad \text { as } \theta \rightarrow 0 .
$$

It follows from here that the error term in (87)

$$
\rho_{n}^{(2)}(\theta) \longrightarrow 0, \quad \text { as } \theta \rightarrow 0
$$

Considering together (81), (87) and (88) and, after some computation, taking into account a continuity property of $\widehat{F}^{\prime \prime}(s)$, we obtain (82).

The Lemma is proved.

With the help of the above established lemmas, we state and prove now the analogue of Law of Large Numbers and the Central Limit Theorem for $S_{n}$.

Theorem 16. Let $\beta<1$ and $\alpha<\infty$. Then

$$
\lim _{n \rightarrow \infty} \mathbb{P}\left\{\frac{S_{n}}{n}<u\right\}= \begin{cases}0, & \text { if } u<1+\gamma \\ 1, & \text { if } u \geq 1+\gamma\end{cases}
$$

where $\gamma=(\alpha-1) /(1-\beta)$.

Proof. Denoting $\psi_{n}(\theta)$ be the Laplace transform of distribution of $S_{n} / n$ it follows from formula (71) that $\psi_{n}(\theta)=T_{n}\left(\theta_{n}\right)$, where $\theta_{n}=\exp \{-\theta / n\}$. The theorem statement is equivalent to that for any fixed $\theta \in \mathbb{R}_{+}$

$$
\psi_{n}(\theta) \longrightarrow e^{-\theta(1+\gamma)}, \quad \text { as } n \rightarrow \infty
$$

From Lemma 10 follows

$$
\ln \psi_{n}(\theta) \sim-\left(1-\frac{u\left(\theta_{n}\right)}{\beta}\right) n+\frac{\beta \gamma(2+\gamma)}{1-\beta} \frac{\theta^{3}}{n^{3}} \sum_{k=0}^{n-1} u^{k}\left(\theta_{n}\right),
$$

as $n \rightarrow \infty$. The first addendum, owing to (80), becomes

$$
\left(1-\frac{u\left(\theta_{n}\right)}{\beta}\right) n \sim(1+\gamma) \theta-\gamma \frac{1+\beta(1+\gamma)}{1-\beta} \frac{\theta^{2}}{n}
$$

And the second one, as it is easy to see, has a decrease order of $\mathscr{O}\left(1 / n^{3}\right)$. Therefore from (90) and (91) follows (89). The Theorem is proved.

We note that in view of the relation (91), it can be estimated the rate of convergence of $S_{n} / n \longrightarrow(1+\gamma)$ as $n \rightarrow \infty$.

Theorem 17. Let $\beta<1, \alpha<\infty$, and $\gamma=(\alpha-1) /(1-\beta)$. Then

$$
\mathbb{P}\left\{\frac{S_{n}-\mathbb{E} S_{n}}{\sqrt{2 \Psi n}}<x\right\} \longrightarrow \Phi(x), \quad \text { as } n \rightarrow \infty,
$$


where the constant

$$
\Psi=\gamma \frac{1+\beta(1+\gamma)}{1-\beta}
$$

and $\Phi(x)$ - the standard normal distribution function.

Proof. This time let $\varphi_{n}(\theta)$ be the characteristic function of distribution of $\left(S_{n}-\mathbb{E} S_{n}\right) / \sqrt{2 \Psi n}$ :

$$
\varphi_{n}(\theta):=\mathbb{E}\left[\exp \frac{i \theta\left(S_{n}-\mathbb{E} S_{n}\right)}{\sqrt{2 \Psi n}}\right]
$$

According to (68) we have

$$
\ln \varphi_{n}(\theta) \sim-(1+\gamma) \frac{i \theta n}{\sqrt{2 \Psi_{n}}}+\ln T_{n}\left(\theta_{n}\right), \quad \text { as } n \rightarrow \infty,
$$

where $\theta_{n}=\exp \{i \theta / \sqrt{2 \Psi n}\}$. Combining (71) and Lemma 10 yields

$$
\ln T_{n}\left(\theta_{n}\right) \sim-\left(1-\frac{u\left(\theta_{n}\right)}{\beta}\right) n+\frac{\beta \gamma(2+\gamma)}{1-\beta} \frac{i \theta^{3}}{(2 \Psi n)^{3 / 2}} \sum_{k=0}^{n-1} u^{k}\left(\theta_{n}\right)
$$

In turn from (80) we have

$$
1-\frac{u\left(\theta_{n}\right)}{\beta} \sim-(1+\gamma) \frac{i \theta}{\sqrt{2 \Psi n}}-\frac{\theta^{2}}{2 n} .
$$

Using relations (93) and (94) in (92) follows

$$
\ln \varphi_{n}(\theta)=-\frac{\theta^{2}}{2}+\mathscr{O}\left(\frac{\theta^{3}}{n^{3 / 2}}\right), \text { as } n \rightarrow \infty .
$$

Hence we conclude that

$$
\varphi_{n}(\theta) \longrightarrow \exp \left\{-\frac{\theta^{2}}{2}\right\}, \quad \text { as } n \rightarrow \infty,
$$

and the theorem statement follows from the continuity theorem for characteristic functions.

\section{Competing interests}

The authors declare that they have no competing interests.

\section{Authors' contributions}

All authors have contributed to all parts of the article. All authors read and approved the final manuscript.

\section{References}

[1] K.B.Athreya and P.E.Ney, Branching processes. Springer, New York, 1972.

[2] M.Dwass, 'The total progeny in a branching process'. Journal in Applied Probability, 6 (1969), 682-686.

[3] T.E.Harris, Theory of Branching stochastic process. MIR, Moscow, 1966. (in Russian)

[4] T.E.Harris, 'Some mathematical models for branching processes'. Proceedings of 2-Berkeley Symposium: Mathematical Statistics and Probability, 1951, 305-328. 
[5] A.A.Imomov, 'Limit properties of transition function of continuous-time Markov Branching Processes'. International Journal of Stochastic Analysis, 2014 (2014), http://dx.doi.org/10.1155/2014/409345, 10 pages.

[6] A.A.Imomov, 'Limit Theorem for the Joint Distribution in the Q-processes', Journal of Siberian Federal University: Math. and Physics, 7(3) (2014), 289-296.

[7] A.A.Imomov, 'On Markov analogue of Q-processes with continuous time'. Theory of Probability and Mathematical Statistics, 84 (2012), 57-64.

[8] A.A.Imomov, 'Some asymptotical behaviors of Galton-Watson branching processes under condition of non-extinctinity of it remote future'. Abstracts of Comm. of 8th Vilnius Conference: Probab. Theory and Math. Stat., Vilnius, Lithuania, p.118 (2002).

[9] P.Jagers, Branching Progresses with Biological applications. Great Britain: John Wiley Sons, Pitman Press, 1975.

[10] A.V.Karpenko and S.V.Nagaev, 'Limit theorems for the total number of descendents for the Galton-Watson branching processes'. Theory of Probability and its Applications, 38 (1994), 433-455.

[11] D.P.Kennedy, 'The Galton-Watson process conditioned on the total progeny'. Journal in Applied Probability, 12 (1975), 800-806.

[12] H.Kesten, P.Ney and F.Spitzer, 'The Galton-Watson process with mean one and finite variance'. Theory of Probability and its Applications, 11(4) (1966), 579-611.

[13] F.C.Klebaner, U.Rösler and S.Sagitov, 'Transformations of Galton-Watson processes and linear fractional reproduction'. Advances in Applied. Probability, 39 (2007), 1036-1053.

[14] V.F.Kolchin, Random mappings. Nauka, Moscow, 1984. (in Russian)

[15] J.Lamperti and P.E.Ney, 'Conditioned branching processes and their limiting diffusions'. Theory of Probability and its Applications, 13 (1968), 126-137.

[16] S.V.Nagaev and R.Muhamedhanova, 'Limit phenomena in branching stochastic processes with discrete time'. In book Limit theorems and statistical conclusions, Editor: S.H.Sirajdinov. Tashkent, 43-89, 1966. (in Russian)

[17] A.G.Pakes, 'Revisiting conditional limit theorems for the mortal simple branching process'. Bernoulli, 5(6) (1999), 969-998.

[18] A.G.Pakes, 'Some limit theorems for the total progeny of a branching process'. Advances in Applied Probability, 3 (1971), 176192.

[19] E.Seneta, 'Functional equations and the Galton-Watson process'. Advances in Applied Probability, 1 (1969), 1-42.

[20] R.S.Slack, 'A branching process with mean one and possible infinite variance'. Wahrscheinlichkeitstheor. und Verv. Geb., 9(2) (1968), 139-145. 